\title{
Bernhard Tuider* \\ Die Sammlung für Plansprachen und das Esperantomuseum der Österreichischen Nationalbibliothek - Geschichte, Bestand und Projekte
}

DOI 10.1515/bfp-2017-0027

Zusammenfassung: Durch eine neunzigjährige Sammeltätigkeit entwickelten sich die Sammlung für Plansprachen und das Esperantomuseum - gegründet 1927 als Internationales Esperanto-Museum - zur weltweit größten Fachbibliothek für Plansprachen und Interlinguistik. Die Sammlung ist nicht nur Bibliothek und Museum, sondern seit ihrer Gründung werden auch Archivalien gesammelt.

Schlüsselwörter: Wien; Österreichische Nationalbibliothek; Plansprachen; Interlinguistik; Fachbibliothek

The Department of Planned Languages and the Esperanto Museum of the Austrian National Library - History, Holdings, and Projects

Abstract: Thanks to ninety years of continuous collecting the Department of Planned Languages and Esperanto Museum - founded in 1927 as the International Esperanto Museum - has become the world's largest specialised library of planned languages and language planning. The Department is not just library and museum, but since its foundation it has also collected archival items.

Keywords: Vienna; Austrian National Library; planned languages; interlinguistics; specialised library

Das Esperantomuseum und die Sammlung für Plansprachen wurden 1927 als privater Verein „Internacia Esperanto-Muzeo en Wien“ gegründet und 1928 als Corpus separatum in die Österreichische Nationalbibliothek integriert. Durch eine neunzigjährige kontinuierliche Sammeltätigkeit - die aus politischen Gründen von 19381945 unterbrochen war - entwickelte sich eine weltweit einzigartige Fachbibliothek für Plansprachen und Interlinguistik, die 1990 die Bezeichnung Sammlung für Plansprachen erhielt.

*Kontaktperson: Mag. Bernhard Tuider, bernhard.tuider@onb.ac.at

\section{Geschichte}

\subsection{Gründung}

Die Gründung des Esperantomuseums erfolgte 1927 durch Hugo Steiner (1878-1969), einem pensionierten Eisenbahnbeamten, der bereits 1911 begonnen hatte, Esperanto zu lernen. Die Idee zu einem Esperantomuseum stammte aber nicht von ihm selbst, sondern von Felix Zamenhof (1868-1933), ${ }^{1}$ der im Sommer 1927 während des 19. Esperanto-Weltkongresses in Danzig den vorausblickenden Vorschlag machte, anlässlich des 50-Jahr-Jubiläums der Sprache Esperanto im Jahr 1937 eine internationale Esperanto-Bibliothek zu gründen.

„Estis la 31. de julio 1927, kiam en kunveno de la reprezentantoj de la Landaj Asocioj dum la XIX-a Univ. Kong. de Esp. en Danzig, kiun mi ĉeestis estante prezidanto de la Aŭstria Esperanto-Asocio, Felikso Zamenhof, la frato de nia neforgesebla [...] Dro Ludoviko Lazaro Zamenhof, faris la proponon, krei okaze de la 50-jara jubileo de Esperanto ie en la mondo Internacian Esperanto-Bibliotekon. [...] Reveninte el Danzig mi tuj ekagis.“2

Hugo Steiner nahm unverzüglich den Impuls von Felix Zamenhof auf, er beabsichtigte aber von Beginn an, nicht nur Bücher und Zeitschriften zu bewahren. Die Esperantobibliothek sollte auch museale und archivalische Funktionen erfüllen, deshalb sammelte er ebenso Fotografien, museale Objekte, Plakate, Verschlussmarken und Archi-

1 Felix Zamenhof ist ein Bruder des Initiators der Sprache Esperanto, Ludwik Zamenhof (1859-1917), der 1887 das erste Esperanto-Lehrbuch „Lingvo Internacia“ veröffentlichte.

2 Steiner (1957) IX. Übersetzung: „Es war der 31. Juli 1927, als bei einem Treffen der Vertreter der Landes-Vereine während des 19. Esperanto-Weltkongresses in Danzig, an dem ich als Präsident der Österreichischen Esperanto-Vereinigung teilnahm, Felix Zamenhof, der Bruder unseres unvergesslichen [...] Dr. Ludwik Lazar Zamenhof, den Vorschlag machte, anlässlich des 50-jährigen Esperanto-Jubiläums irgendwo auf der Welt eine internationale Esperanto-Bibliothek einzurichten. [...] Aus Danzig zurückgekehrt, machte ich mich sofort ans Werk.“ 
valien. Außerdem erfasste Hugo Steiner nicht nur Literatur in bzw. über Esperanto, sondern allgemein Materialien zum Thema Plansprachen und Interlinguistik. Dennoch wählte er den Namen „Internationales Esperantomuseum" unter der Annahme, dass ca. 90 Prozent des Bestandes Esperanto betreffen würden. Dem Namen nach handelte es sich somit um ein Dokumentationszentrum zum Thema Esperanto, de facto war das Esperantomuseum aber von Beginn an Museum, Bibliothek und Archiv für Plansprachen, was Hugo Steiner retrospektiv folgendermaßen kommentierte:

„Ŝajnis al mi, ke la fondo de simpla biblioteko ne sufiĉus, kaj mi decidis starigi pli ampleksan Instituton [sic] kun pli vaste fiksitaj celoj, nome kolekti kaj konservi la tutan materialon, ligitan kun la historia movado de Esperanto kaj de la mondhelplingvaj sistemoj kaj mondlingvaj problemoj sur scienca bazo kaj dokumente pruvi la disvastiĝon de Esperanto en la tuta mondo.“3

Nach dem Ersten Weltkrieg begann eine Blütezeit des Esperanto in Österreich - wie auch in vielen anderen europäischen Ländern. Dementsprechend günstig waren 1927 die Voraussetzungen für die Gründung eines Esperantomuseums: 1922 gab es in Wien mehr als 70 Esperanto-Kurse, die hauptsächlich von Polizisten, Arbeitern und Katholiken organisiert wurden, ${ }^{4} 1924$ kamen 3400 Teilnehmer zum 16. Esperanto-Weltkongress nach Wien und 1925 erfolgte in der Bundeshauptstadt der 5. Kongress der „Sennacieca Asocio Tutmonda“. Ein Teilnehmer dieses Kongresses war auch der spätere Bürgermeister von Wien und Österreichische Bundespräsident Franz Jonas (1899-1974), der bereits 1922 begonnen hatte, Esperanto zu lernen und ab 1926 für mehrere Jahre die sozialdemokratische Esperanto-Zeitschrift „La socialisto“ redigierte.

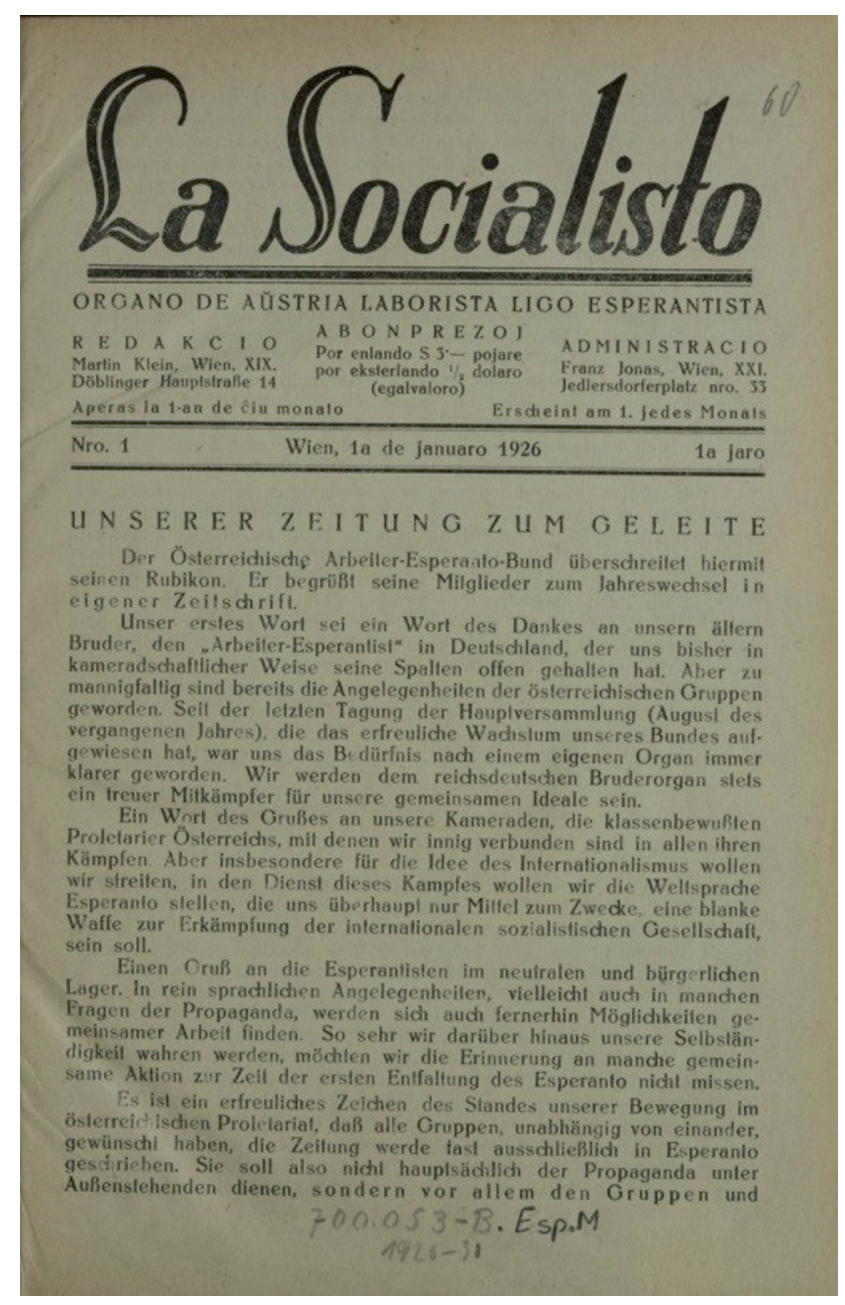

Abb. 1: La socialisto, 1926

Auch konservative Politiker schätzten die Vorteile und den Nutzen des Esperanto, ein besonderer Förderer der Sprache war Johann Schober (1874-1932). ${ }^{5}$ Johann Schober beteiligte sich in den 1920er-Jahren aktiv am „EsperantoLeben “, ${ }^{6}$ er unterstützte Esperanto durch seine politische Autorität, und dadurch lernte er auch Hugo Steiner kennen. In einer biografischen Notiz machte Hugo Steiner sogar darauf aufmerksam, dass Johann Schober sowohl bei der Gründung des Esperantomuseums als auch bei dessen Integration in die Österreichische Nationalbiblio-

3 Steiner (1957) IX. Übersetzung: „Es schien mir, dass die Gründung einer einfachen Bibliothek nicht genügen würde, und so entschloss ich mich, ein umfangreicheres Institut mit weiter gesteckten Zielen ins Leben zu rufen, nämlich das gesamte Material zu sammeln und zu bewahren, das in Verbindung mit der historischen Esperanto-Bewegung und mit den Systemen von Welthilfssprachen und dem Sprachenproblem steht, und so auf wissenschaftlicher Basis durch Dokumentation die Verbreitung des Esperanto auf der ganzen Welt zu beweisen."

4 Vgl. Plakat (1922).
5 Anm. Johann Schober hatte in den 1920er-Jahren mehrere politische Ämter inne; u. a. war er Präsident der Wiener Polizei (1918-1932), Mitbegründer und erster Präsident der Interpol (1923-1932), Unterrichtsminister (1929), Handelsminister (1930), Justizminister (1930/ 31), Außenminister (1921/22, 1929/30 und 1930-1932) und Bundeskanzler (1921/22 und 1929/30) der Republik Österreich.

6 Anm. Johann Schober war Teilnehmer des 7. Österreichischen Esperantokongress (1927), den er auch präsidierte. 


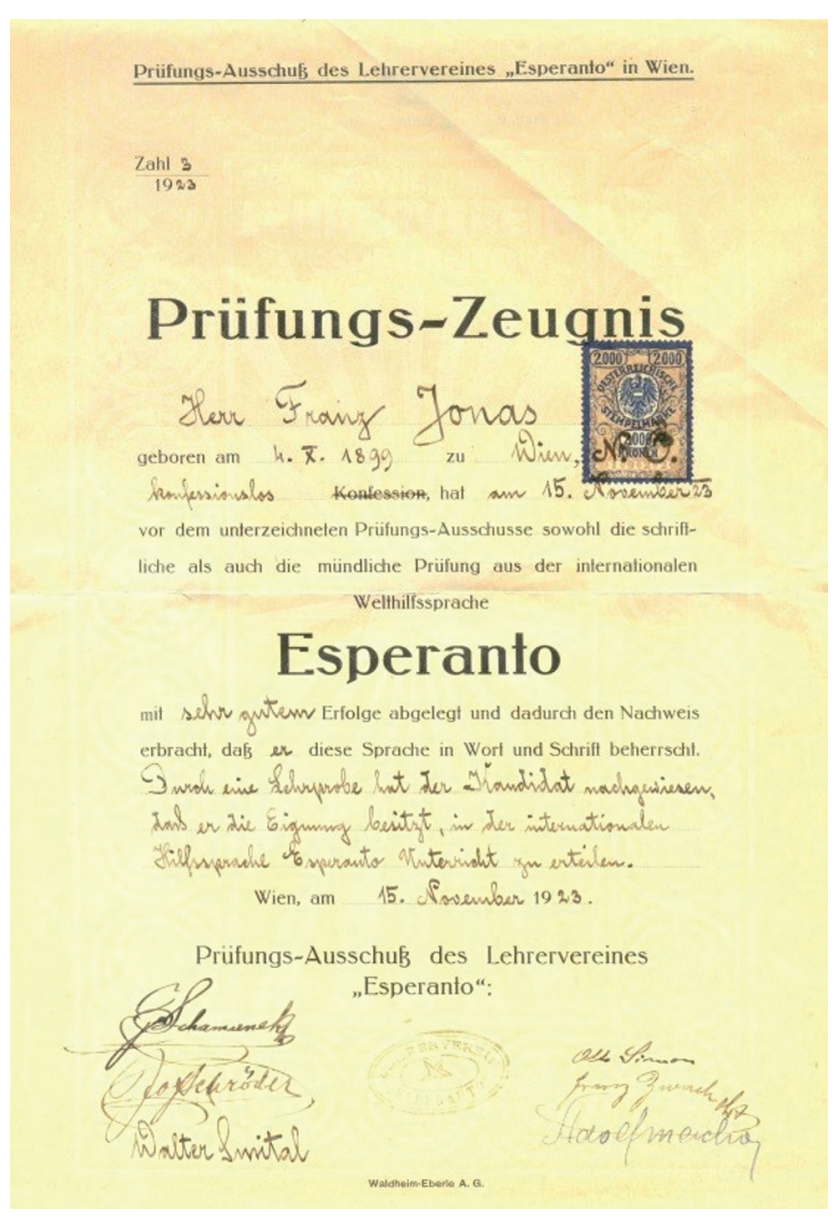

Abb. 2: Prüfungszeugnis von Franz Jonas für die Lehrbefähigung in Esperanto, 1923

thek die entscheidende Kraft war. ${ }^{7}$ Zudem erhielt Hugo Steiner Unterstützung von einem weiteren prominenten Politiker, Ignaz Seipel (1876-1932), dem er unmittelbar nach seiner Rückkehr aus Danzig über sein Bestreben berichtete, eine internationale Esperantobibliothek gründen zu wollen.

Aufgrund dieser Kontakte zu einflussreichen Persönlichkeiten des öffentlichen Lebens erhielt Hugo Steiner in einer Zeit äußerster Raumknappheit zunächst provisorische Räumlichkeiten im Kellergeschoß des Ministeriums für Land- und Forstwirtschaft in der Liebiggasse 5. Im Juni 1928 erfolgte die Übersiedlung in renovierte Stallräume des Klein-Mariazellerhofes in der Annagasse 5, dem damaligen Sitz des Rechnungshofes.

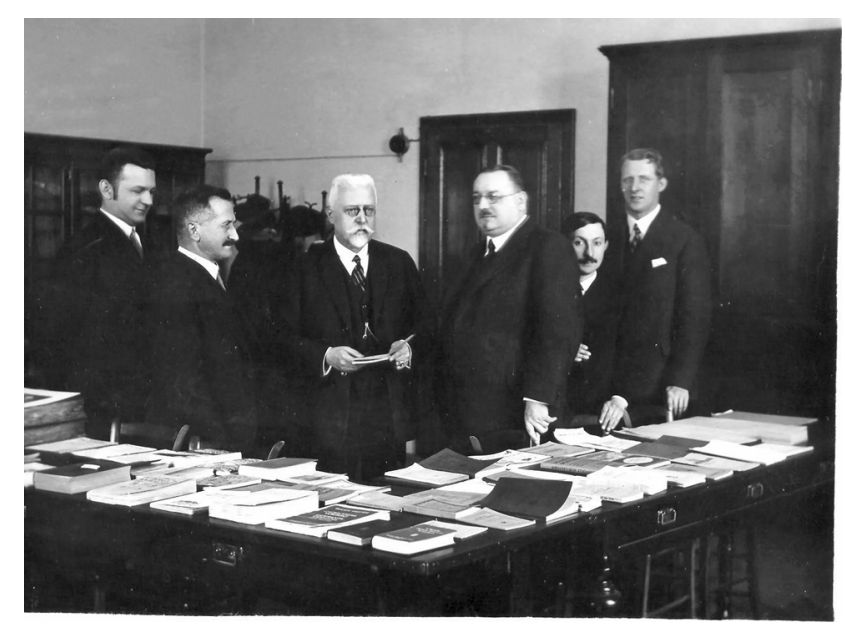

Abb. 3: Hugo Steiner (2 v. l.), Johann Schober (3 v. l.) und Josef Bick (4 v. I.) im Esperantomuseum, Wien 1931

\subsection{Schließung durch die Gestapo und Wiedereröffnung nach dem Zweiten Weltkrieg}

Die feierliche Eröffnung des Internationalen Esperantomuseums, bei der ca. 600 Gäste aus mehr als 30 Ländern anwesend waren, fand am 1 . August 1929 im Prunksaal der Österreichischen Nationalbibliothek statt. ${ }^{8}$

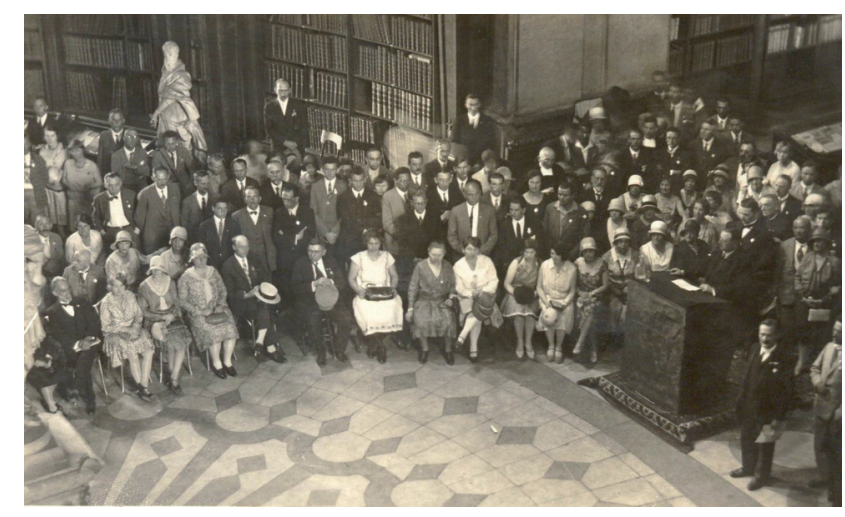

Abb. 4: Eröffnung des Internationalen Esperantomuseums, Wien 1929
7 Steiner (1938) 7: „1927 begann ich mit den Vorarbeiten zur Gründung des Internationalen Esperanto-Museums in Wien, dem einzigen in der Welt. Ich fand hiebei größte Förderung durch den damaligen Polizeipräsidenten Schober und dann besonders als er Bundeskanzler war.“
8 Teilnehmer der Eröffnungsfeier waren u. a. Lidia Zamenhof (19041942), Julia Isbrücker (1887-1971), Odo Bujwid (1857-1942), Hugo Steiner und der Präsident der Republik Österreich Wilhelm Miklas (1872-1956). 


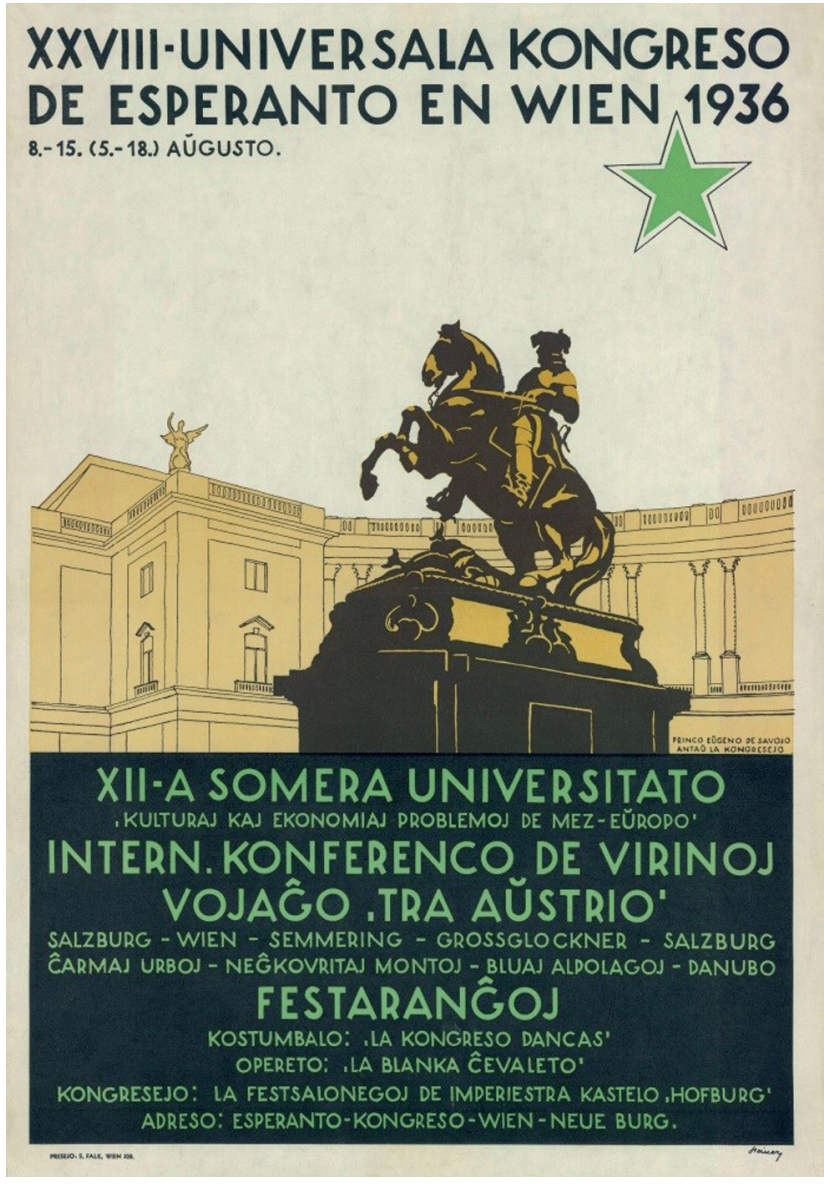

Abb. 5: 28. Esperanto-Weltkongress, Wien 1936

Im Zuge der Integration in die Österreichische Nationalbibliothek erfolgte am 1. Oktober 1929 die Übersiedlung des Museums in das Abraham-à-Santa-Clara-Zimmer im Augustinerstöckl. Im Juni 1930 konnten großzügigere Räumlichkeiten im zweiten Geschoß der Neuen Hofburg am Heldenplatz bezogen werden, wo das Museum und die Bibliothek bis zur Schließung im März 1938 untergebracht waren.

„La 11-an de marto 1938 Steiner devis paroli en Radio-Wien, sed la saman tagon jam estis enmarŝantaj la trupoj de Hitler. Mi skribis al la Ĝeneraldirektoro de Nacia Biblioteko, ke mi konfidis s-anon Reg. Kons. Fritz STENGEL anstataŭi min kaj duontage ankoraŭ laboris en la Muzeo ĝis la 20.IV., por ordigi la kancelarion. Kiam mi la 20.IV. posttagmeze venis en la Muzeon, ĝi estis fermata de GESTAPO.“9

9 Steiner (1957) XXIX. Übersetzung: „Am 11. März 1938 musste Steiner in Radio Wien sprechen, aber am selben Tag befanden sich die HitlerTruppen auf dem Einmarsch. Ich schrieb an den Generaldirektor der Nationalbibliothek, dass ich [...] Regierungsrat Fritz STENGEL meine Vertretung anvertraut hatte, und ich arbeitete bis zum 20. IV. noch halbtags im Museum, um die Kanzlei zu ordnen. Als ich am 20. IV. am
Nachdem die Gestapo das Museum geschlossen und versiegelt hatte, konfiszierten die Nationalsozialisten das Einlaufbuch sowie den Zettelkatalog des Esperantomuseums und beabsichtigten alle plansprachlichen Bücher und Dokumente nach Berlin zu transportieren, da das Reichsicherheitshauptamt (RSHA) plante, das gesamte Esperantomuseum sicherstellen zu lassen. Der kommissarische Leiter der Nationalbibliothek, Paul Heigl (1887-1945), konnte aber den Abtransport verhindern durch einen Brief, in dem er darauf hinwies, dass es sich bei dem plansprachlichen Material um das Eigentum der Österreichischen Nationalbibliothek handle, das Wien nicht verlassen dürfe.

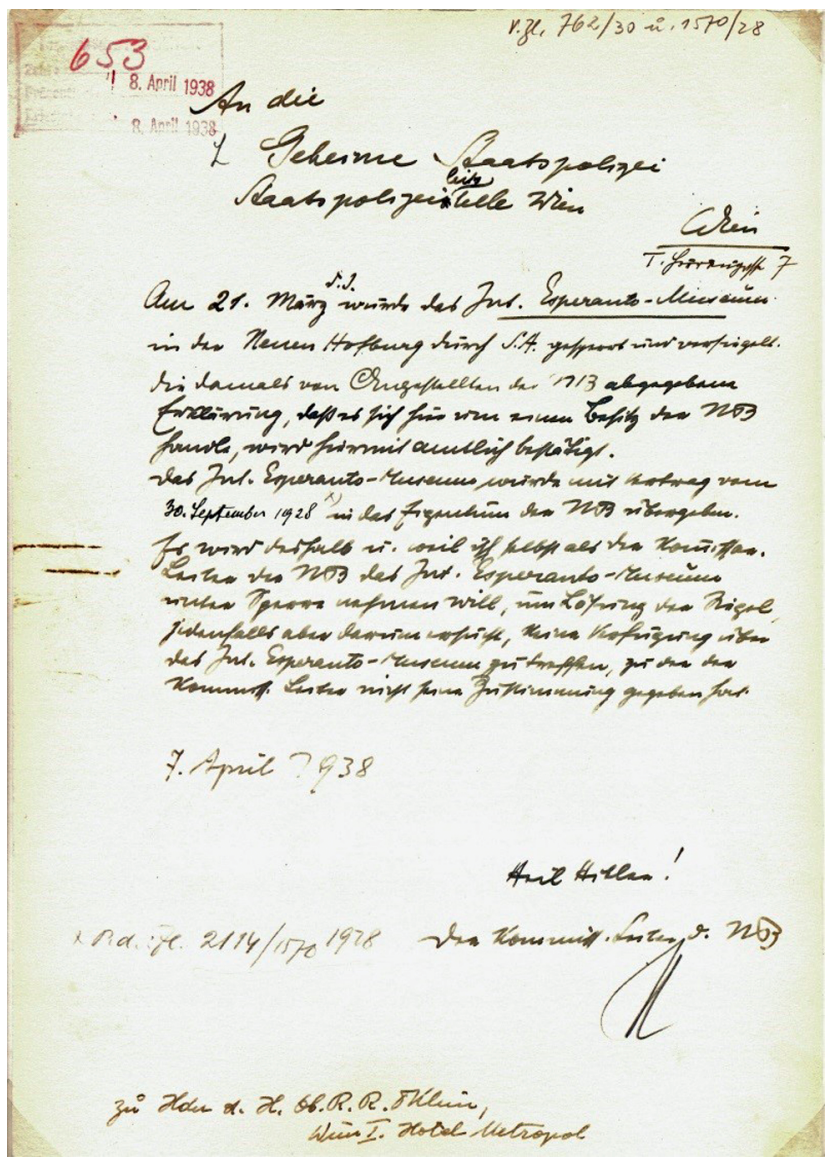

Abb. 6: Brief von Paul Heigl an die Gestapo, Wien 1938

Die Dokumente und Bücher - die Sammlung umfasste zu diesem Zeitpunkt ca. 3000 Werke - wurden deshalb in Kisten verpackt und unzugänglich in den Kellern der Hofburg aufbewahrt, wo sie den Krieg unbeschadet überstanden. ${ }^{10}$

Nachmittag ins Museum kam, fand ich es von der Gestapo geschlossen vor."

10 Vgl. Köstner (2005) 180 ff. 
Nach der Wiedereröffnung des Esperantomuseums im Michaelertrakt der Wiener Hofburg 1947 konnte - im Gegensatz zu anderen Esperanto-Sammlungen, die während des Nationalsozialismus zerstört worden waren - auf dem erhalten gebliebenen Bestand aufgebaut werden. Während die räumlichen und klimatischen Verhältnisse am neuen Standort relativ ungünstig waren, gab es in der Nachkriegszeit auch Fortschritte, da nun zwei von der Österreichischen Nationalbibliothek zur Verfügung gestellte Bibliothekare in der Sammlung beschäftigt waren: ab 1958 Walter Hube (1923-2000), der nach dem Tod von Hugo Steiner im Jahr 1969 die Leitung übernahm, und ab 1966 Herbert März (1929-1995).

Eine wesentliche Leistung nach dem Zweiten Weltkrieg war der kontinuierliche und systematische Bestandsaufbau - 1967 umfasste die Fachbibliothek bereits 14500 Bände, ${ }^{11} 199320000$ Bände ${ }^{12}$-, die öffentliche Wirkung der Sammlung war allerdings relativ gering. Bis in die 1980er-Jahre existierte kein Ausstellungskonzept, zudem fehlten finanzielle Mittel für eine Modernisierung des Museums. Diese prekäre Situation begann sich erst gegen Ende der 1980er-Jahre zu ändern, als Herbert Mayer Direktor des Esperantomuseums wurde.

\subsection{Modernisierungen im 21. Jahrhundert}

Mit der Bestellung von Dr. Johanna Rachinger zur Generaldirektorin der Österreichischen Nationalbibliothek im Juni 2001 begann auch für das Esperantomuseum und die Sammlung für Plansprachen eine neue Ära. Johanna Rachinger erkannte sofort die Defizite und leitete deshalb 2002 die Übersiedlung der Bibliothek und des Museums in das Palais Mollard-Clary in der Herrengasse 9 ein, die 2005 ihren Abschluss fand.

Die neue Unterbringung an einem zentralen Standort eröffnete der Einrichtung völlig neue Chancen, die einer Neugründung gleichkamen. Die verkehrstechnisch günstigere Lage, ein modernes Museumskonzept und zeitgemäße Präsentationsmethoden führten zu einem sprunghaften Ansteigen der Besucherzahlen: Waren es im Jahre 2004 noch 3098, so kamen 2006 bereits 5025 Besucher in das Esperantomuseum.

Auch das Problem des mangelnden Magazinraums wurde durch die Übersiedlung nachhaltig und sehr zufriedenstellend gelöst. Das Magazin hat nun eine Größe von ca. $180 \mathrm{~m}^{2}$ und erfüllt alle aktuellen Standards auf den

11 Vgl. Hube (1967) 153.

12 Vgl. Österreichische Nationalbibliothek (1994) 77.

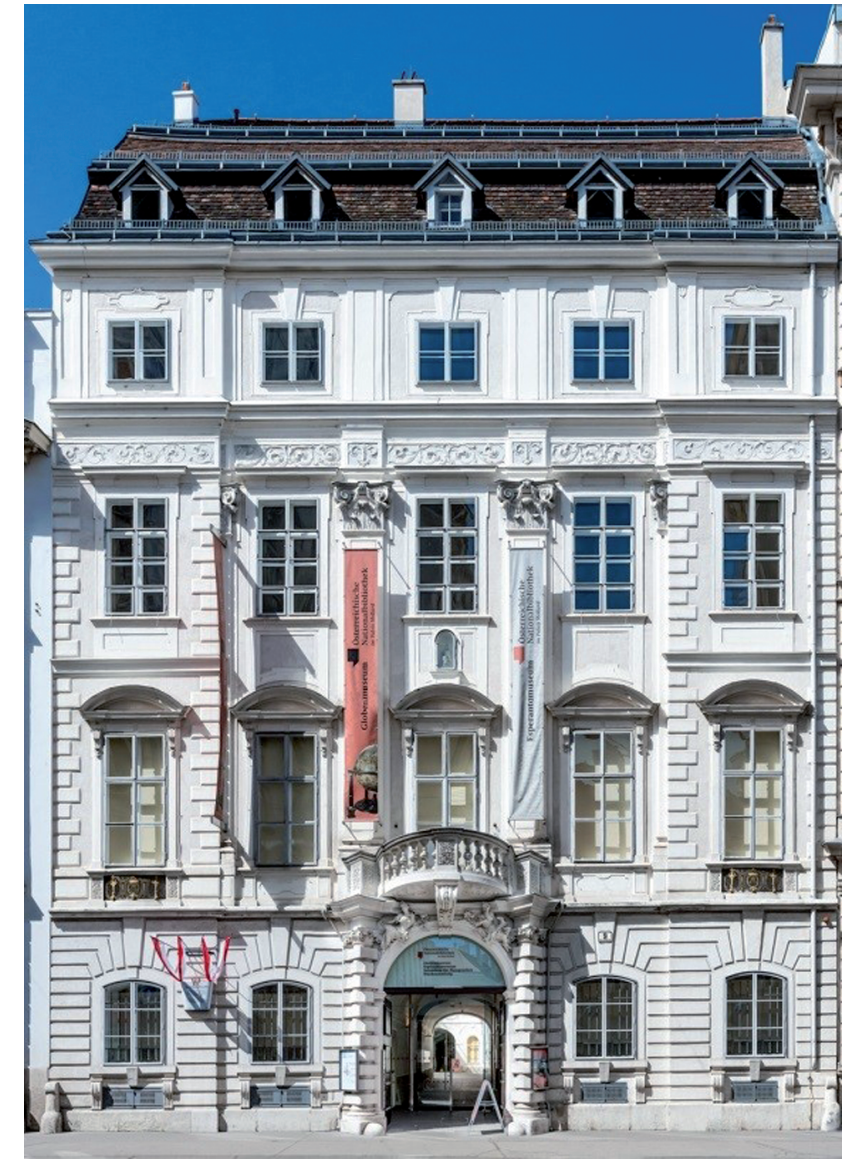

Abb. 7: Palais Mollard-Clary, Herrengasse 9, 1010 Wien

Gebieten der Sicherheit, Lagerung und Konservierung. Es bietet einen großzügigen Raum für den gesamten Bestand der Sammlung für Plansprachen und des Esperantomuseums, der folgende Medien umfasst, ca.:

- 40000 Flugblätter

- 35000 Bibliotheksbände

- 25000 Zeitungsausschnitte

- 22000 Fotos und Fotonegative

- 10000 Manuskripte und Autografen

- 3700 verschiedene Zeitungs- und Zeitschriftentitel

- 3000 museale Objekte

- 1500 Plakate

- 850 audiovisuelle Materialien

\section{Die moderne Bibliothek}

Als Teil der Österreichischen Nationalbibliothek ist die Sammlung für Plansprachen ein dienstleistungsorientiertes Informations- und Forschungszentrum, das alle Anforderungen einer modernen Bibliothek erfüllt. Zu den konkreten Zielen der Sammlung gehören u. a. das: 
- Erweitern des Bestandes durch kontinuierliches und systematisches Sammeln

- Konservieren und Bewahren des plansprachlichen Materials

- elektronische Katalogisieren des gesamten Bestandes

- öffentliche Zur-Verfügung-Stellen plansprachlicher Dokumente

- schriftliche und mündliche Beantworten von Anfragen

- Erforschen und Veröffentlichen von plansprachlichem Material

Diese Ziele werden durch mehrere Projekte realisiert, insbesondere durch Katalogisierungs- und Digitalisierungsprojekte.

\subsection{Katalogisierungsprojekte}

\subsubsection{Der Bibliothekskatalog - Trovanto}

Das Ziel der Katalogisierungsprojekte ist die vollständige elektronische Katalogisierung des Sammlungsbestandes und das Zur-Verfügung-Stellen der Kataloge über das Internet. Diese Projekte inkludieren auch zum Teil - bezüglich der Fotos, der Ansichtskarten, der Plakate und musealen Objekte - die Präsentation von bereits digitalisierten Beständen im Internet.

1996 begann die elektronische Katalogisierung der Bücher, Zeitungen und Zeitschriften, 2005 wurde die retrospektive Katalogisierung abgeschlossen und im gleichen Jahr erfolgte die Migration der Katalogisate in das neue Bibliothekssystem Aleph. Dadurch kann seit nunmehr 20 Jahren vollkommen orts- und zeitunabhängig über den OPAC Trovanto im Sammlungsbestand recherchiert werden. Trovanto - der Katalog der Sammlung für Plansprachen und des Esperantomuseums - ist ein Teil des Gesamtkataloges der Österreichischen Nationalbibliothek, des sogenannten QuickSearch. Zugänglich ist Trovanto u. a. über die Webseite der Sammlung für Plansprachen, die nicht nur in Deutsch und Englisch, sondern auch in Esperanto abrufbar ist.

Die Verschlagwortung im Katalog Trovanto erfolgt in Deutsch und in Esperanto, somit sind in beiden Sprachen Recherchen möglich. Durch die Integration des Bildkataloges - Bildarchiv Austria - kann seit 2013 über eine einzige Datenbank nach allen katalogisierten Materialien der Sammlung, in mehr als 50000 Katalogisaten, recherchiert werden, nicht nur nach Druckschriften, sondern auch nach CDs, DVDs, Tonbändern, Bildmaterialien, wie Fotos, Ansichtskarten, Verschlussmarken, musealen Objekten sowie
Plakaten, und Archivalien. ${ }^{13}$ Die Katalogisate der Bildmaterialien enthalten sowohl schriftliche Informationen zu den Bildobjekten als auch eine Ansicht des jeweiligen Digitalisates. Außerdem haben die Datensätze von mehr als 700 Büchern ${ }^{14}$ und 70 verschiedenen Zeitschriftentiteln ${ }^{15}$ einen direkten Link zum Digitalisat des Werkes, so dass Bibliotheksbenutzer, die eine dieser Druckschriften lesen möchten, von jedem Internet-Arbeitsplatz - auch außerhalb der Österreichischen Nationalbibliothek - virtuell in den bereits digitalisierten Druckwerken blättern können.

Dieser Service zeigt, wie sehr sich die Benutzungsmöglichkeiten innerhalb der letzten 20 Jahre gewandelt haben. Mussten Leser in den 1990er-Jahren Literatur noch mit relativ großem Zeitaufwand in Zettelkatalogen suchen und sich die Bücher ausgeben lassen, erfolgen Recherche und Bestellung heute komfortabel über das Internet. Durch eine ständig wachsende Zahl von digitalisierten Dokumenten ist oft nicht einmal mehr der Weg in die Bibliothek unbedingt notwendig, um zu den gesuchten Informationen zu kommen, da direkt in Trovanto Digitalisate geöffnet und eingesehen werden können.

Leser, die noch nicht digitalisierte Sammlungsbestände nutzen möchten, können diese über den Katalog Trovanto bestellen und anschließend im Leseraum der Sammlung für Plansprachen lesen bzw. im Falle von audiovisuellen Materialien abspielen.

Um eine verbesserte Suchbarkeit von in Sammelbänden publizierten Aufsätzen zu gewährleisten, werden seit 2006 im Rahmen des Projekts IV-Scan die Datensätze von Sammelbänden durch gescannte Inhaltsverzeichnisse und bibliographische Details angereichert. Dadurch können Leser das Inhaltsverzeichnis eines Sammelbandes direkt vom Katalogisat aus öffnen und einsehen. Da im Rahmen des Projekts IV-Scan auch Katalogisate der einzelnen Artikel systematisch erstellt werden, sind nun auch Texte in Aufsatzsammlungen, Festschriften, Tagungsberichten und Ausstellungskatalogen in Trovanto recherchierbar und somit für Leser leichter zugänglich.

13 Unter den Archivalien, die in der Sammlung für Plansprachen bewahrt werden, befinden sich auch 56 Vor- und Nachlässe (Stand: April 2017), zu den umfangreichsten gehören jene von Kálmán Kalocsay (1891-1976), Eugen Wüster (1898-1977), Walter Mudrak (1904-1998), Andreas Juste (1918-1998), Jacques Bol (1922-2009), Otto Back $\left(1926^{\star}\right)$ und Manuel de Seabra (1932*).

14 Bei den mehr als 900 gescannten Büchern (Stand: April 2017) handelt es sich vor allem um „Esperanto-Frühdrucke“, die vor 1910 publiziert wurden.

15 Insgesamt erfolgte die Digitalisierung von ca. 700 Jahrgängen (Stand: April 2017) aus dem Zeitraum von 1889 bis 1946. 


\subsubsection{Die Bilddatenbank - Bildarchiv Austria}

Seit dem Jahr 2007 ist ein großer Teil der 22000 Fotografien der Sammlung für Plansprachen in der Bilddatenbank der Österreichischen Nationalbibliothek - dem Bildarchiv Austria - systematisch katalogisiert und gescannt worden. Zudem erfolgte auch eine umfangreiche Digitalisierung und Katalogisierung von Ansichtskarten, Verschlussmarken, musealen Objekten und Plakaten, so dass sich momentan alleine aus der Sammlung für Plansprachen mehr als 17000 Katalogisate in der Datenbank befinden, von denen mehr als 12000 über das Internet recherchierbar sind. Neuerwerbungen der Sammlung werden kontinuierlich katalogisiert, dementsprechend wächst die Zahl der Katalogisate der Bilddatenbank - wie auch des Katalogs Trovanto - kontinuierlich.

\subsection{Digitalisierungsprojekte}

Der Schwerpunkt der Digitalisierungsprojekte liegt bei den Esperanto-Zeitschriften, den frühen Esperanto-Publikationen und den Esperanto-Tonbändern. Analoge Tonträger werden in absehbarer Zeit nicht mehr abspielbar sein, folglich sind v. a. die Digitalisierung und Langzeitarchivierung der Tondokumente sehr wichtige Aufgaben. Da Esperanto weltweit von einer beachtlichen Sprachgemeinschaft gesprochen wird, ${ }^{16}$ ist das Digitalisieren und Zur-VerfügungStellen von Dokumenten über das Internet ein sehr bedeutendes und weitreichendes Bibliotheksservice.

Mit den Projekten zur Bestandsdigitalisierung werden mehrere Ziele verfolgt: Sie sollen einerseits die Sichtbarkeit und Zugänglichkeit der Bestände verbessern, indem die Digitalisate über das Internet recherchierbar werden. Andererseits dienen die Digitalisierungen dem Schutz des Bestandes, indem gefährdete Objekte nach der digitalen Reproduktion aus dem Publikumsverkehr genommen und somit geschont werden können. Ein weiterer großer Vorteil von Digitalisierungen ist die uneingeschränkte Öffnungszeit des virtuellen Lesesaals, der an sieben Tagen pro Woche 24 Stunden geöffnet hat und kontinuierlich durch neue Reproduktionen erweitert wird. Die Möglichkeiten des Internets bewirken somit eine massive Änderung in den Nutzungsstrategien, einen umfangreichen, ständig wachsenden virtuellen Lesesaal mit permanenter Öff-

16 Im Januar 2016 haben mehr als 300000 Personen in Facebook angegeben, Esperanto zu sprechen. Auf der Lernplattform www.ler nu.net haben seit Dezember 2002 mehr als 225000 Personen begonnen, Esperanto zu lernen, auf der Webseite www.duolingo.com seit Mai 2015 mehr als 820000 Personen (Stand: April 2017). nungszeit und folglich eine Förderung und potenzielle Intensivierung der wissenschaftlichen Forschung. Benutzer haben aber nicht nur die Möglichkeit, im virtuellen Lesesaal in den Digitalisaten zu blättern, sondern sie können auch durch „Digitalisierungen on Demand“ von jedem Objekt - sofern es der konservatorische Zustand erlaubt eine digitale Reproduktion für eine zeitgemäße Verwendungsform am Computer bestellen.

In Zusammenarbeit mit der Abteilung für Digitale Services der Österreichischen Nationalbibliothek realisierte die Sammlung für Plansprachen seit dem Jahr 2006 mehrere Digitalisierungsprojekte, dementsprechend kann bereits ein großer Teil des Sammlungsbestandes über das Internet recherchiert und im virtuellen Lesesaal genutzt werden. Bis April 2017 erfolgte die Digitalisierung von ca.:

- 900 Büchern

- 70 Periodika (insgesamt ca. 700 Jahrgänge)

- 7300 Fotografien

- 2200 Ansichtskarten

- 2500 musealen Objekten

- 800 Plakaten

War der Bestand der Sammlung für Plansprachen und des Esperantomuseums bis zum Ende des 20. Jahrhunderts nur in einem eingeschränkten Maß und für jene Personen wahrnehmbar, die das Museum in Wien besuchten, verleiht die durch systematische Digitalisierungen verbesserte öffentliche Präsenz der Sammlung für Plansprachen und dem Esperantomuseum erstmals in ihrer Geschichte eine weitreichende „äußerliche“ Sichtbarkeit, die ihrem inneren Wert entspricht.

\section{Das Esperantomuseum}

Das Esperantomuseum der Österreichischen Nationalbibliothek ist weltweit eines der ältesten Sprachmuseen und eine der bedeutendsten Einrichtungen ihrer Art. Das $\mathrm{Mu}$ seum ist fachlich, administrativ und räumlich eng mit der Sammlung für Plansprachen verbunden, in der insgesamt rund 500 plansprachliche Projekte dokumentiert werden.

Im Esperantomuseum werden die Sprache Esperanto und Themen aus der Geschichte der Esperantobewegung präsentiert, aber auch allgemein das Verhältnis vom Menschen zur Sprache und zum Sprachenproblem thematisiert. Das Museum veranschaulicht, dass Plansprachen - von Menschen nach bestimmten Kriterien bewusst geschaffene Sprachen - in spezifischen historischen und kulturellen Kontexten entstehen und es vermittelt dadurch auch, dass Esperanto ein kulturhistorisches Phänomen und die Mani- 


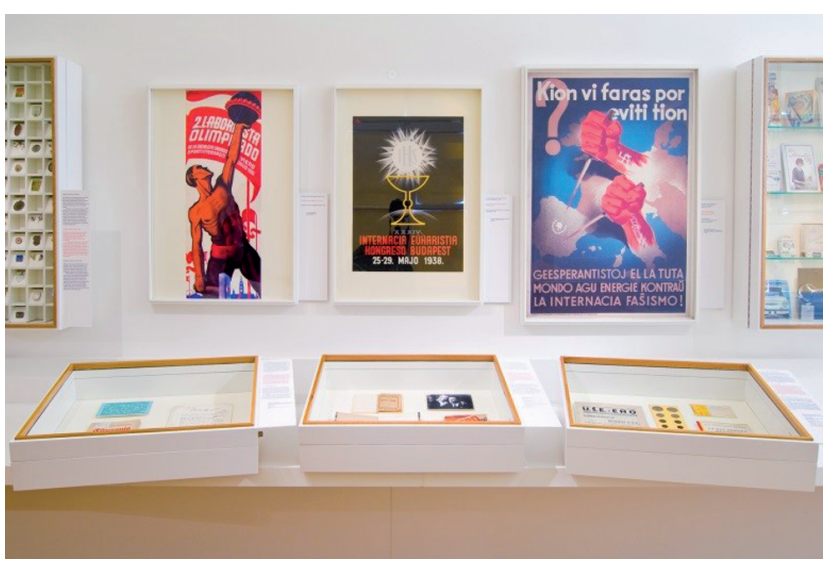

Abb. 8: Esperantomuseum, 2017

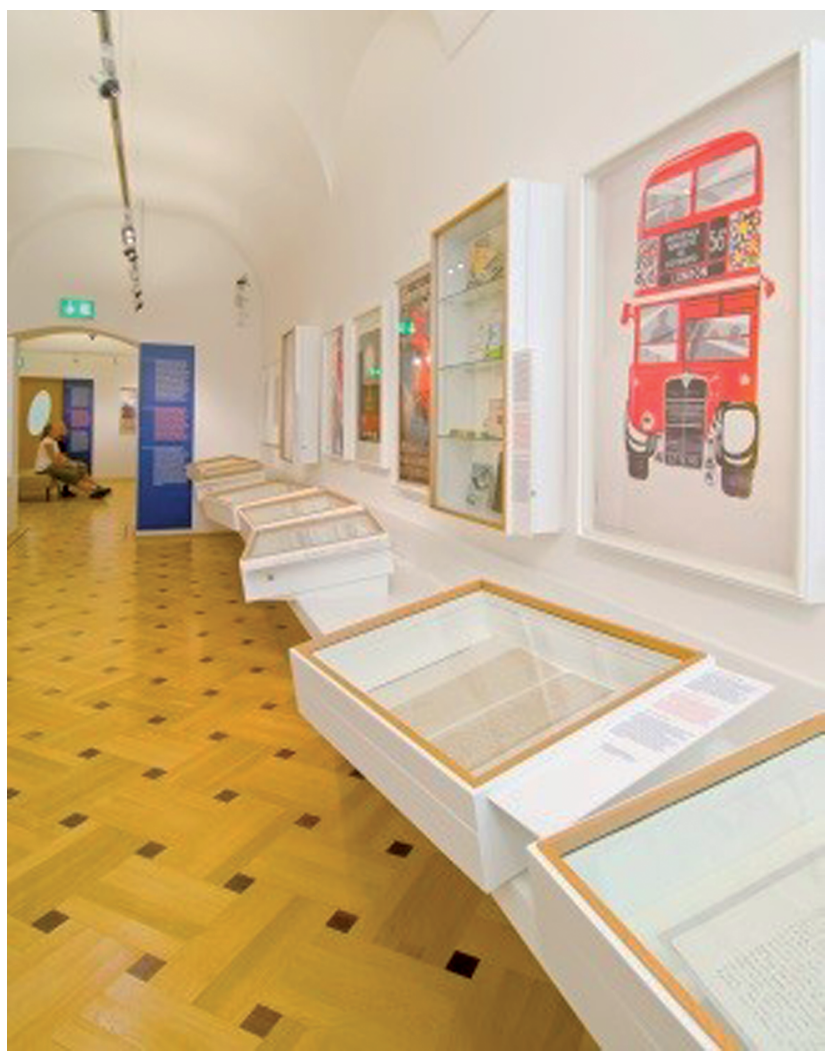

Abb. 9: Esperantomuseum, 2017

festation einer speziellen sprachlichen Kreativität ist. Die Ausstellung folgt nicht einer Metaerzählung, sondern es werden aus einer Vielfalt an Narrativen, die in der Vergangenheit im Kontext mit Plansprachen entstanden sind, exemplarisch, anhand von ausgewählten Objekten einige besondere Aspekte hervorgehoben. Im Zentrum steht dabei die erfolgreichste bewusst geschaffene Sprache: Esperanto. Interaktive Medienstationen, bedeutende Publikationen, Objekte, Plakate und Archivalien präsentieren eindrucksvoll die weite Verbreitung und vielseitige Ver- wendung der Sprache von der Veröffentlichung des „Unua Libro“ 1887 bis zur Literaturproduktion und den Esperanto-Weltkongressen in der Gegenwart. Ein kurzer Film vermittelt einen Überblick über die Geschichte, die Grammatik und die gegenwärtige Praxis der Sprache und ein Video-Kurs der BBC vermittelt einen Eindruck, wie gesprochenes Esperanto klingt. Zudem können Besucher durch audiovisuelle Vermittlung eine Vielfalt an Plansprachen kennenlernen, wie z.B. Volapük, Ido und Interlingua. Klangvoll bietet das Esperantomuseum auch so unterschiedliche Projekte wie die Lingua Ignota der Hildegard von Bingen (1098-1179), Solresol von Jean François Sudre (1787-1862), die Neuslawische Sprache von Ignaz Hošek (1852-1919) oder Klingonisch von Marc Okrand (1948*).

\section{Literatur}

Hube, Walter (1967): 40 Jahre Internationales Esperanto-Museum. In: Biblos. Österreichische Zeitschrift für Buch- und Bibliothekswesen, Dokumentation, Bibliographie und Bibliophilie, 16 (3), 149-53.

Köstner, Christina (2005): Glück im Unglück. Das Esperantomuseum an der Nationalbibliothek Wien 1938-1945. In: Language Problems \& Language Planning, 29 (2), 177-86.

Österreichische Nationalbibliothek (Hrsg.) (1994): Jahresbericht 1993. Wien: Österreichische Nationalbibliothek.

Österreichische Nationalbibliothek (Hrsg.) (2015): Jahresbericht. 1976-2015. Wien: Österreichische Nationalbibliothek.

Plakat (1922): Wegweiser für Unterricht und Fortbildung in Esperanto. Anfängerkurse und Übungszirkel in Wien. Signatur: ÖNB ESP Pla-5-207.

Steiner, Hugo (1938): Mein Leben und Wirken (unveröffentlichtes Typoskript in der Sammlung für Plansprachen).

Steiner, Hugo (1957): Katalogo pri la kolektoj de Internacia EsperantoMuzeo en Wien. Parto I.: Sistema katalogo pri la Esperantopresajoj laŭ la Internacia Dekuma Klasifiko. Wien: Internacia Esperanto-Muzeo.

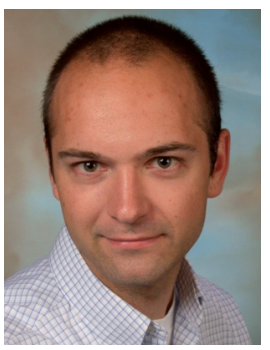

Mag. Bernhard Tuider

Teamleiter Sammlung für Plansprachen und Esperantomuseum der Österreichischen Nationalbibliothek

Palais Mollard Herrengasse 9 A-1010 Wien Österreich bernhard.tuider@onb.ac.at 